\title{
Measuring CP violation by low-energy medium-baseline neutrino oscillation experiments *
}

\author{
Hisakazu Minakata ${ }^{1,2}$ and Hiroshi Nunokawa ${ }^{3}$ \\ ${ }^{1}$ Department of Physics, Tokyo Metropolitan University \\ 1-1 Minami-Osawa, Hachioji, Tokyo 192-0397, Japan, and \\ ${ }^{2}$ Research Center for Cosmic Neutrinos, Institute for Cosmic Ray Research, \\ University of Tokyo, Kashiwa, Chiba 277-8582, Japan \\ ${ }^{3}$ Instituto de Física Gleb Wataghin, Universidade Estadual de Campinas, UNICAMP \\ 13083-970 - Campinas, Brazil.
}

\begin{abstract}
In this talk we discuss the possibility to measure $\mathrm{CP}$ violation in neutrino oscillation experiments using the neutrino beam with energy which is lower $\left(E_{\nu} \gtrsim 100 \mathrm{MeV}\right)$ than the one usually considered (typically $>1 \mathrm{GeV}$ ) in accelerator experiments. The advantage of using such lower energy neutrino beam is that despite the smaller detection cross sections, the effect of $\mathrm{CP}$ violation is larger and the optimal length of baseline can be rather short, $30-50 \mathrm{~km}$, being free from matter effect contamination.
\end{abstract}

Very impressive results in atmospheric neutrino observation from the SuperKamiokande experiment 22 and the persistent discrepancy between the observed and the calculated flux of solar neutrinos 3] provide the strongest indications for neutrino oscillations induced by neutrino masses and lepton flavor mixing. Determination of all the mixing parameters, in particular the $\mathrm{CP}$ violating Kobayashi-Maskawa phase [4], is one of the most challenging goals in particle physics.

In this talk, we consider the possibility of measuring the effect of leptonic $\mathrm{CP}$ violation (or equivalently $\mathrm{T}$ violation) in neutrino oscillation [5 9] experiments within the standard three neutrino flavor framework. We will end up with the proposal of medium baseline experiments with much lower energy $\left(E_{\nu} \gtrsim 100 \mathrm{MeV}\right)$ neutrino beam than the ones usually considered (typically $E_{\nu}>1 \mathrm{GeV}$ ) in accelerator experiments.

Let us consider the neutrino mass squared differences suggested by the atmospheric neutrino observation [2] and the MSW solutions [10] to the solar neutrino problem. In the standard threeflavor neutrino mixing scheme, all the independent mass squared differences are exhausted if we assume the oscillation interpretations of the atmospheric as well as solar neutrino data. They are, from the atmospheric neutrinos $\Delta m_{13}^{2} \approx$ $\Delta m_{23}^{2}=\Delta m_{\text {atm }}^{2} \simeq(2-5) \times 10^{-3} \mathrm{eV}^{2}$ and from

*Talk presented by H. Nunokawa based on the work in Ref.[1] at "NuFact'00" workshop, Monterey, CA, USA, May 22-26, 2000. the solar neutrino data, $\Delta m_{12}^{2}=\Delta m_{\text {solar }}^{2} \simeq(4-$ $10) \times 10^{-6} \mathrm{eV}^{2}$ (SMA), $(2-20) \times 10^{-5} \mathrm{eV}^{2}(\mathrm{LMA})$ or $(6-20) \times 10^{-8} \mathrm{eV}^{2}(\mathrm{LOW})$ where SMA, LMA, and LOW denote the small mixing angle, large mixing angle and the low $\Delta m^{2}$ MSW solutions, respectively 11, 12. We use the notation $\Delta m_{i j}^{2} \equiv m_{j}^{2}-m_{i}^{2}$ in this report.

Many authors including us examined the question of how to separate the genuine $\mathrm{CP}$ violating effect due to the leptonic Kobayashi-Maskawa phase from the fake one induced by matter effect [7] 9]. Here we take a simple alternative strategy to look for a region of parameters in which the matter effect is "ignorable" in the first approximation.

Let us define the flavor mixing matrix $U$ as $\nu_{\alpha}=U_{\alpha i} \nu_{i}$, where $\nu_{\alpha}(\alpha=e, \mu, \tau)$ and $\nu_{i}(i=$ $1,2,3)$ stand for the gauge and the mass eigenstates, respectively. We take for convenience the representation of $U$ as

$U=e^{i \lambda_{7} \theta_{23}} \Gamma_{\delta} e^{i \lambda_{5} \theta_{13}} e^{i \lambda_{2} \theta_{12}}$

where $\lambda_{i}$ are SU(3) Gell-Mann's matrix and $\Gamma_{\delta}=\operatorname{diag}\left(1,1, e^{i \delta}\right)$ with $\delta$ being the $\mathrm{CP}$ violating phase.

Let us first neglect the possible matter effect and consider the $\mathrm{CP}$ violating effect in vacuum. Under the mass difference hierarchy $\Delta m_{13}^{2} \gg$ $\Delta m_{12}^{2}$ which is implied by the solar and atmospheric neutrino data, the neutrino oscillation 
probability in vacuum can be written as

$$
\begin{aligned}
P\left(\nu_{\beta}\right. & \left.\rightarrow \nu_{\alpha}\right)=4\left|U_{\alpha 3}\right|^{2}\left|U_{\beta 3}\right|^{2} \sin ^{2}\left(\frac{\Delta_{13} L}{2}\right) \\
& =-4 \operatorname{Re}\left[U_{\alpha 1} U_{\alpha 2}^{*} U_{\beta 1}^{*} U_{\beta 2}\right] \sin ^{2}\left(\frac{\Delta_{12} L}{2}\right) \\
& -2 J \sin \left(\Delta_{12} L\right)\left[1-\cos \left(\Delta_{13} L\right)\right] \\
& +4 J \sin \left(\Delta_{13} L\right) \sin ^{2}\left(\Delta_{12} L\right),
\end{aligned}
$$

where $\Delta_{i j} \equiv \frac{\Delta m_{i j}^{2}}{2 E}$ and $L$ is the distance traveled by neutrinos. Here the effect of CP violation comes in through $J$ in eq. (2) which is defined as

$J_{\alpha \beta ; i, j} \equiv \operatorname{Im}\left[U_{\alpha i} U_{\alpha j}^{*} U_{\beta i}^{*} U_{\beta j}\right]$

as it is the unique (in three-flavor mixing scheme) measure for CP violation as first observed by Jarlskog [13] in the case of quark mixing. With our parametrization of mixing matrix, it takes the form $J= \pm c_{12} s_{12} c_{23} s_{23} c_{13}^{2} s_{13} \sin \delta$, where the sign is positive for $(e, \mu)$ and $(1,2)$ and $+(-)$ corresponds to their (anti-) cyclic permutations of $(\alpha, \beta)$ and $(i, j)$.

We first note that from the expression of $J$, if any one of the mixing angles is extremely small or very close to $\pi / 2$ there is little hope in detecting the $\mathrm{CP}$ violation even if $\delta$ is large. For this reason, we will not deal with the case of SMA MSW solar neutrino solution.

We also notice that the effect of $\mathrm{CP}$ violation is larger if the quantity

$$
\begin{aligned}
\Delta_{12} L= & 0.026\left(\frac{\Delta m_{12}^{2}}{10^{-5} \mathrm{eV}^{2}}\right)\left(\frac{L}{100 \mathrm{~km}}\right) \\
& \left(\frac{E}{100 \mathrm{MeV}}\right)^{-1},
\end{aligned}
$$

is larger. From this we see that larger values of $\Delta m_{12}^{2}$, lower $E_{\nu}$ and larger $L$ is preferrable. For this reason we consider largest plausible value of $\Delta m_{12}^{2}$ coming from LMA MSW solution (which is chosen among the suggested solutions) to the solar neutrino problem and lowest possibly practical energy $\sim 100 \mathrm{MeV}$ which can be provided by accelerator experiments. We note that larger distance $L$ is preferrable just in terms of the probability but it has to be determined such that the observables of the $\mathrm{CP}$ violating effect which is proportional to the expected number of events (not the probability itself) is significant. (See our results below).

Next let us consider the matter effect. We first note that at neutrino energy of $\sim 100 \mathrm{MeV}$ there is a hierarchy among the relevant energy scales;

$$
\begin{aligned}
& \Delta_{13} \equiv \frac{\Delta m_{13}^{2}}{2 E} \sim 10^{-11} \mathrm{eV} \\
& \gg a \sim \frac{\Delta m_{12}^{2}}{2 E} \equiv \Delta_{12} \sim 10^{-13} \mathrm{eV},
\end{aligned}
$$

where we assume $\rho=2.7 \mathrm{~g} / \mathrm{cm}^{3}$ and $\Delta m_{12}^{2} \sim$ $(3.0-4.0) \times 10^{-5} \mathrm{eV}^{2} \sin ^{2} 2 \theta_{12} \sim 0.8$ assuming LMA MSW solution [1] and $\Delta m_{13}^{2} \sim \times 10^{-3} \mathrm{eV}^{2}$ $\sin ^{2} 2 \theta_{23} \sim 1$ assuming $\nu_{\mu}-\nu_{\tau}$ atmospheric neutrino oscillation solution.

Thanks to the mass hierarchy (5) we can formulate the perturbation theory and it is straightforward to compute the neutrino oscillation probabilities $P\left(\nu_{\mu} \rightarrow \nu_{e}\right)$ as well as $P\left(\bar{\nu}_{\mu} \rightarrow \bar{\nu}_{e}\right)$ under the adiabatic approximation [14,15,1]. For example, the appearance probability $P\left(\nu_{\mu} \rightarrow \nu_{e}\right)$ reads

$$
\begin{aligned}
& P\left(\nu_{\mu} \rightarrow \nu_{e}\right)=4 s_{23}^{2} c_{13}^{2} s_{13}^{2} \sin ^{2}\left(\frac{1}{2} \Delta_{13} L\right) \\
& \quad+c_{13}^{2} \sin 2 \theta_{12}^{M}\left[\left(c_{23}^{2}-s_{23}^{2} s_{13}^{2}\right) \sin 2 \theta_{12}^{M}\right. \\
& \left.\quad+2 c_{23} s_{23} s_{13} \cos \delta \cos 2 \theta_{12}^{M}\right] \\
& \times \sin ^{2}\left[\frac{1}{2} \xi \Delta_{12} L\right]-2 J_{M}\left(\theta_{12}^{M}, \delta\right) \sin \left[\xi \Delta_{12} L\right],
\end{aligned}
$$

with

$\sin 2 \theta_{12}^{M} \equiv \sin 2 \theta_{12} / \xi$

where

$\xi \equiv \sqrt{\left(\cos 2 \theta_{12}-\frac{a}{\Delta_{12}} c_{13}^{2}\right)^{2}+\sin ^{2} 2 \theta_{12}}$

and $J_{M}$ is the matter enhanced Jarlskog factor, $J_{M}\left(\theta_{12}^{M}, \delta\right)=\cos \theta_{12}^{M} \sin \theta_{12}^{M} c_{23} s_{23} c_{13}^{2} s_{13} \sin \delta$, and we have averaged the rapidly oscillating piece driven by $\Delta_{13}$ in the $\mathrm{CP}$ violating term. The antineutrino transition probability $P\left(\bar{\nu}_{\mu} \rightarrow \bar{\nu}_{e}\right)$ is given by the same expressions as above but replacing $a$ and $\delta$ by $-a$ and $-\delta$, respectively.

Let us note that if $L<1,000 \mathrm{~km}$ or so, the approximation $\sin \left(\xi \Delta_{12} L\right) \simeq \xi \Delta_{12} L$ is valid and the expressions of the oscillation probabilities approximately reduce to those in the vacuum because

$\sin 2 \theta_{12}^{M}\left(\right.$ or, $\left.\bar{\theta}_{12}^{M}\right) \xi \Delta_{12}=\sin 2 \theta_{12} \Delta_{12}$.

Using the mixing parameters described in the caption of Fig. 1, we verify by our numerical computations that the matter effect in fact cancels out. In Fig. 11 we plot the oscillation probabilities for neutrino and anti-neutrino, and their difference, $\Delta P \equiv P\left(\nu_{\mu} \rightarrow \nu_{e}\right)-P\left(\overline{\nu_{\mu}} \rightarrow \overline{\nu_{e}}\right)$, as a function of distance from the source with the neutrino energy $E=60 \mathrm{MeV}$, for both $\nu_{\mu} \rightarrow \nu_{e}$ and 
$\overline{\nu_{\mu}} \rightarrow \overline{\nu_{e}}$, where it corresponds to the resonance energy in the neutrino channel. Although we sit on at the resonance energy of neutrino channel the features in the resonant neutrino flavor conversion cannot be traced in Fig. 11, but rather we observe the one very much similar to the vacuum oscillation even for $L$ as large as $1000 \mathrm{~km}$.
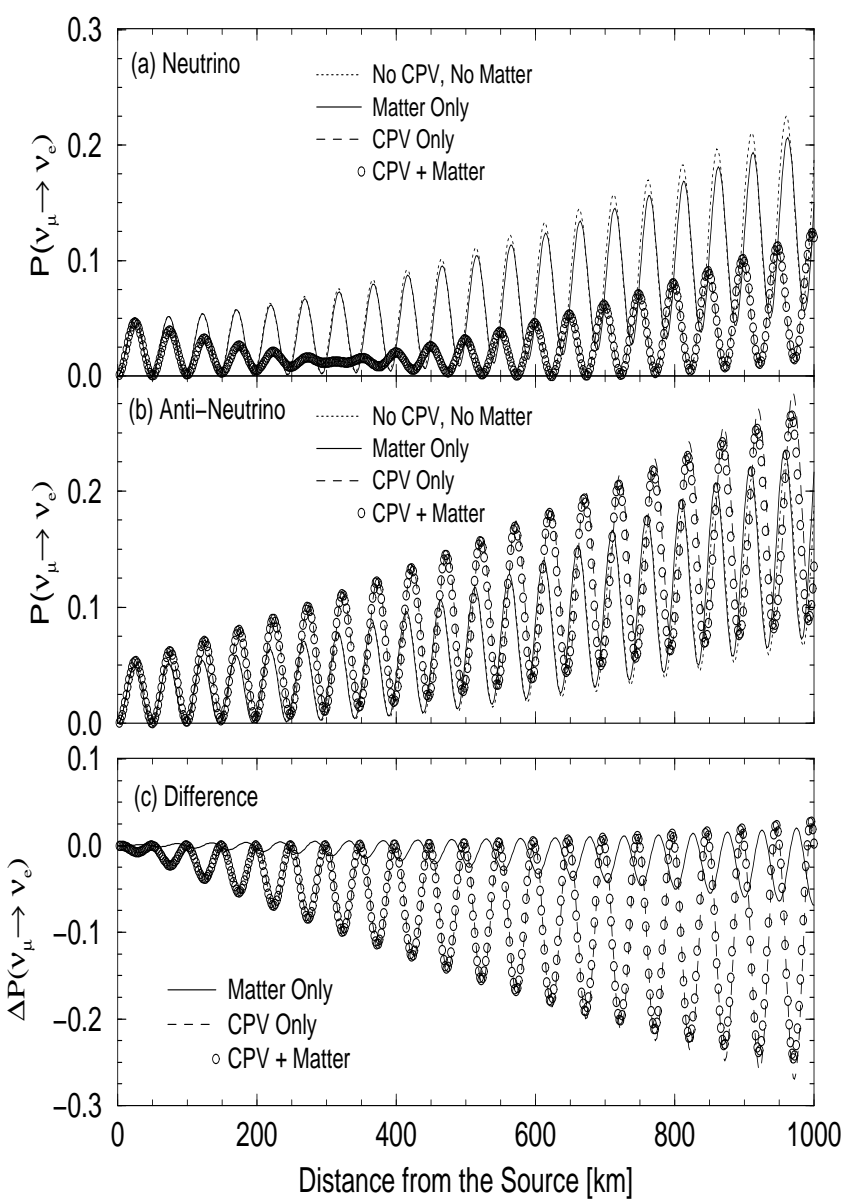

Figure 1. Oscillation probability for (a) neutrinos, $P\left(\nu_{\mu} \rightarrow \nu_{e}\right)$, (b) anti-neutrinos, $P\left(\bar{\nu}_{\mu} \rightarrow \bar{\nu}_{e}\right)$, and (c) their difference, $\Delta P\left(\nu_{\mu} \rightarrow \nu_{e}\right) \equiv P\left(\nu_{\mu} \rightarrow\right.$ $\left.\nu_{e}\right)-P\left(\bar{\nu}_{\mu} \rightarrow \bar{\nu}_{e}\right)$ with fixed neutrino energy $E_{\nu}=$ $60 \mathrm{MeV}$, are plotted as a function of distance from the source. The mixing parameters are fixed to be $\Delta m_{13}^{2}=3 \times 10^{-3} \mathrm{eV}^{2}, \sin ^{2} 2 \theta_{23}=1.0, \Delta m_{12}^{2}=$ $2.7 \times 10^{-5} \mathrm{eV}^{2}, \sin ^{2} 2 \theta_{12}=0.79, \sin ^{2} 2 \theta_{13}=0.1$ and $\delta=\pi / 2$. We take the matter density as $\rho=2.72 \mathrm{~g} / \mathrm{cm}^{3}$ and the electron fraction as $Y_{e}=$ 0.5 .

Now we discuss the possible experiments which utilize the imitating vacuum mechanism to measure leptonic CP violation. Measurement of $\mathrm{CP}$ violation at a few \% (or smaller) level at neu- trino oscillation experiments at $E \simeq 100 \mathrm{MeV}$ leaves practically the unique channel $\nu_{\mu} \rightarrow \nu_{e}$. Experimentally, what is relevant is the the number of events not the oscillation probability itself. Here, as a measure of CP violation, we consider the ratio of the expected number of events due to $\nu_{\mu} \rightarrow \nu_{e}$ and $\overline{\nu_{\mu}} \rightarrow \overline{\nu_{e}}$ reactions.

In such low energy appearance experiment we have the following difficulties; (1) smaller cross sections (2) lower flux due to larger beam opening angle, $\Delta \theta \simeq 1(\mathrm{E} / 100 \mathrm{MeV})^{-1}$ radian. Therefore, we are invited to the idea of the baseline as short as possible, because the luminosity decreases as $L^{-2}$ as baseline length grows. However, from Fig. 1 we see that longer the baseline, the larger the $\mathrm{CP}$ violating effect and it is expected that there exist some optimal distance which gives the signal most (statistically) significant.

Detection of low-energy neutrinos at better than a few \% level accuracy requires supermassive detectors. Probably the best thinkable detection apparatus is the water Cherenkov detector of SuperKamiokande type. Let us estimate the expected number of events at a megaton detector placed at $L=100 \mathrm{~km}$. We assume that the neutrino beam flux 10 times as intense as (despite the difference in energy) that of the design luminosity in $\mathrm{K} 2 \mathrm{~K}$ experiment [17]. We note that in the future it is expected that even an 100 times more intense proton flux than KEK-ps seems possible at Japan Hadron Facility 18].

The dominant $\nu_{e}$-induced reaction in water at around $E=100 \mathrm{MeV}$ is not the familiar $\nu_{e}-e$ elastic scattering but the reaction on ${ }^{16} O, \nu_{e}^{16} O \rightarrow e^{-} F \quad 19$. The cross section of the former reaction is about $\sigma\left(\nu_{e} e \rightarrow \nu_{e} e\right)=$ $0.93 \times 10^{-42}\left(\frac{E}{100 \mathrm{MeV}}\right) \mathrm{cm}^{2}$, while the latter is $\sigma\left(\nu_{e}^{16} O \rightarrow e^{-} F\right) \simeq 10^{-39} \mathrm{~cm}^{2}$ at $E=100$ $\mathrm{MeV}$ [19], which is a factor of 1000 times larger. However, since the number of oxygen in water is $1 / 10$ of the number of electrons, net the number of events due to the reaction $\nu_{e}^{16} O \rightarrow e^{-} F$ is larger than that of $\nu_{e} e$ elastic scattering by a factor of 100. The neutrino flux at the detector located at $L=250 \mathrm{~km}$ is, by our assumption, 10 times more intense than the neutrino flux at SuperKamiokande in K2K experiment. The latter is, roughly speaking, $3 \times 10^{6}\left(\frac{\mathrm{POT}}{10^{20}}\right) \mathrm{cm}^{-2}$ where POT stands for proton on target. Therefore, the expected number of events $N$ assuming 
$100 \%$ conversion of $\nu_{\mu}$ to $\nu_{e}$ is given by

$N \simeq 6300\left(\frac{L}{100 \mathrm{~km}}\right)^{-2}\left(\frac{V}{1 \mathrm{Mton}}\right)\left(\frac{\mathrm{POT}}{10^{21}}\right)$

In the antineutrino channel, the dominant reaction is $\bar{\nu}_{e} p \rightarrow e^{+} n$ with cross section $\sigma \simeq$ $0.4 \times 10^{-39} \mathrm{~cm}^{2}$ at $E=100 \mathrm{MeV}$. The event number due to this reaction, assuming the same flux of $\bar{\nu}_{\mu}$ as $\nu_{\mu}$, is similar to that of (10) because the cross section is about half but there are two free protons per one oxygen. There is additional oxygen reaction $\bar{\nu}_{e}^{16} O \rightarrow e^{+} N$ with approximately factor 3 smaller cross section than that of $\nu_{e}^{16} O$ 19 .

In order to estimate the optimal distance, we compute the expected number of events in neutrino and anti-neutrino channels as well as their ratios as a function of distance taking into account of neutrino beam energy spread. For definiteness, we assume that the average energy of neutrino beam $\langle E\rangle=100 \mathrm{MeV}$ and beam energy spread of Gaussian type with width $\sigma_{E}=10$ $\mathrm{MeV}$. We present our results in Fig. 2.

We see from this plot that taking the error (only statistical one for simplicity) into account, $L$ must not be too short or too long and there exist on optimal distance which is rather short, $L \sim 30-50 \mathrm{~km}$, much shorter than that of the current (or future) long-baseline neutrino oscillation experiments (see Fig. 2). At such distance the significance of the $\mathrm{CP}$ violating signal is as large as 3-4 $\sigma$.

We conclude that the experiment is quite feasible under such intense neutrino beam and a megaton detector. Fortunately, the possibility of constructing a megaton water Cherenkov detector is already discussed by the experimentalists [20].

Some final remarks are in order.

1) For the other MSW solutions such as SMA or LOW to the solar neutrino problem, due to the much smaller mixing angle and/or $\Delta m^{2}$, with the same experimental conditions, the expected number of events is smaller than at least a factor of 100 and therefore, the experiment does not appear feasible with the same apparatus.

2) An alternative way of measuring $\mathrm{CP}$ violation is the multiple detector method [7] which may be inevitable if either one of $\nu$ or $\bar{\nu}$ beam is difficult to prepare. It utilizes the fact that the first, the second, and the third terms in the oscillation probability (6) have different $L$ dependences, $\sim L$-independent (after averaging over energy spread of the neutrino beam), $\sim L^{2}$, and
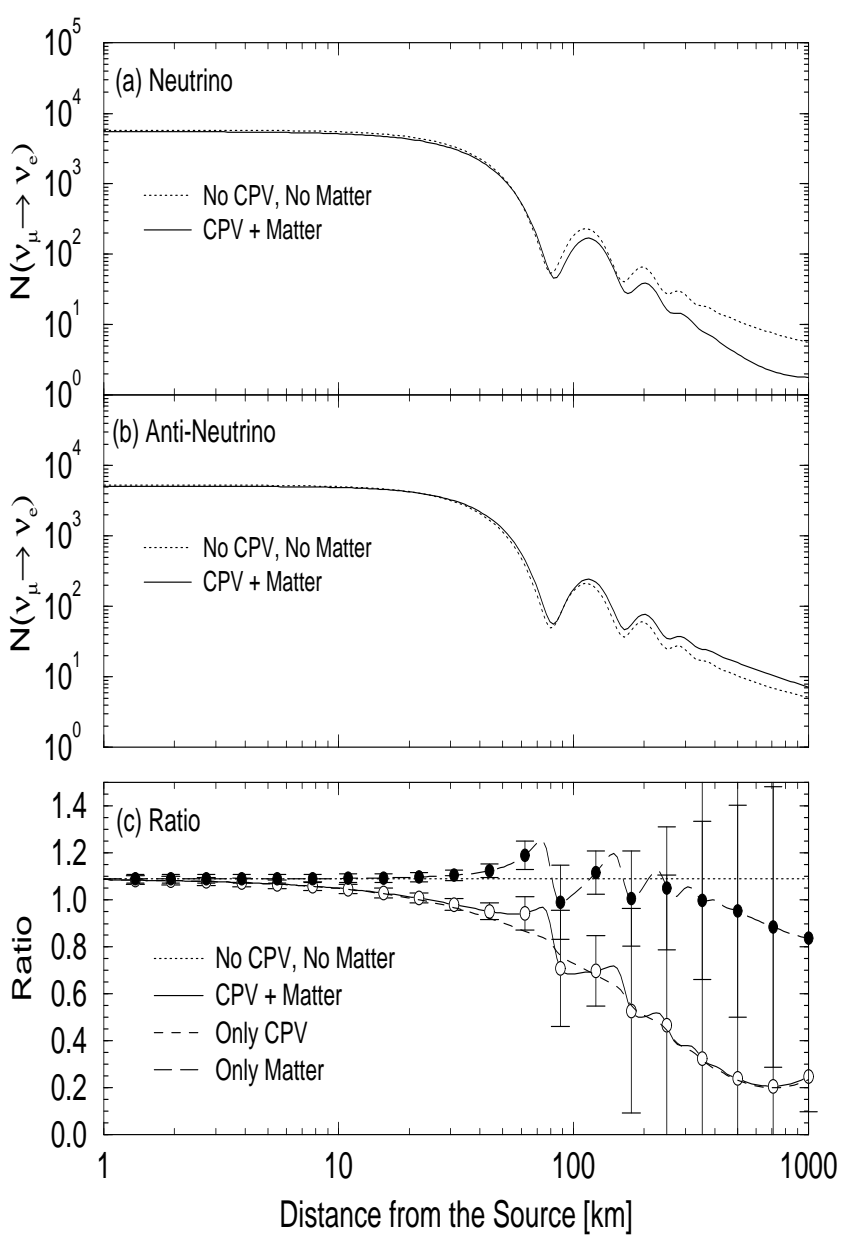

Figure 2. $\quad$ Expected number of events for (a) neutrinos, $N\left(\nu_{\mu} \rightarrow \nu_{e}\right)$, (b) anti-neutrinos, $N\left(\bar{\nu}_{\mu} \rightarrow \bar{\nu}_{e}\right)$, and (c) their ratio $R \equiv N\left(\nu_{\mu} \rightarrow\right.$ $\left.\nu_{e}\right) / N\left(\bar{\nu}_{\mu} \rightarrow \bar{\nu}_{e}\right)$ with a Gaussian type neutrino energy beam with $\left\langle E_{\nu}\right\rangle=100 \mathrm{MeV}$ with $\sigma=10$ $\mathrm{MeV}$ are plotted as a function of distance from the source. Neutrino fluxes are assumed to vary as $\sim 1 / L^{2}$ in all the distance range we consider. The mixing parameters as well as the electron number density are fixed to be the same as in Fig. 1. The error bars are only statistical.

$\sim L$, respectively, in the linear approximation.

3) Intense neutrino beams from muon storage ring at low energies, proposed as PRISM, PhaseRotation Intense Secondary Mesons [21], could be an ideal source for neutrinos for the experiment discussed in this report. Of course, it requires identification of $\overline{\nu_{e}}$ from $\nu_{e}$ by some methods, e.g., by adding Chlorine $\left({ }^{35} \mathrm{Cl}\right)$ into the Water Cherenkov detector to make it sensitive to the characteristic $\sim 8 \mathrm{MeV} \gamma$ rays arising from the absorption of neutron into the Chlorine followed 
by $\bar{\nu}_{e} p \rightarrow e^{+} n$ reaction.

4) We would like to urge experimentalists to think more about the better supermassive detection apparatus than water Cherenkov for highly efficient and accurate measurement of low energy neutrinos.

See Ref. [1] for more detailed discussions on this work.

\section{Acknowledgments}

We thank Takaaki Kajita, Masayuki Nakahata and Kenzo Nakamura for informative discussions on detection of low energy neutrinos. This work was supported by the Brazilian funding agency Fundação de Amparo à Pesquisa do Estado de São Paulo (FAPESP), and by the Grantin-Aid for Scientific Research in Priority Areas No. 11127213, Japanese Ministry of Education, Science, Sports and Culture.

\section{REFERENCES}

1. H. Minakata and H. Nunokawa, hepph/004114.

2. Y. Fukuda et al. (Kamiokande collaboration), Phys. Lett. B335 (1994) 237; Y. Fukuda et al. (SuperKamiokande collaboration), Phys. Rev. Lett. 81 (1998) 1562; T. Kajita, (SuperKamiokande collaboration), Nucl. Phys. (Proc. Suppl.) B77 (1999) 123.

3. Homestake Collaboration, K. Lande et al., Astrophys .J. 496 (1998) 505 ; SAGE Collaboration, J. N. Abdurashitov et al., Phys. Rev. C 60 (1999) 055801; GALLEX Collaboration, W. Hampel et al., Phys. Lett. B 447 (1999) 127; Kamiokande Collaboration, Y. Fukuda et al. Phys. Rev. Lett. 77 (1996) 1683; SuperKamiokande Collaboration, Y. Fukuda et al., Phys. Rev. Lett. 81 (1998) 1158; ibid. 81 (1998) 4279; ibid. 82 (1999) 2430; ibid. 82 (1999) 1810.

4. M. Kobayashi and T. Maskawa, Prog. Theor. Phys. 49 (1973) 652.

5. N. Cabbibo, Phys. Lett. B72, (1978) 333; V. Barger, K. Wisnant and R. J. N. Phillips, Phys. Rev. Lett. 45 (1980) 2084; S. Pakvasa, in Proceedings of the XXth International Conference on High Energy Physics, edited by L. Durand and L. G. Pondrom, AIP Conf. Proc. No. 68 (AIP, New York, 1981), Vol. 2, pp. 1164 .

6. T. K. Kuo and J. Pantaleone, Phys. Lett. B198 (1987) 406.
7. H. Minakata and H. Nunokawa, Phys. Lett. B413 (1997) 369.

8. H. Minakata and H. Nunokawa, Phys. Rev. D57 (1998) 4403.

9. J. Arafune and J. Sato, Phys. Rev. D55 (1997) 1653; J. Arafune, M. Koike and J. Sato, Phys. Rev. D56 (1997) 3093; Erratum ibid., D 60 (1999) 119905; M. Tanimoto, Phys. Rev. D55 (1997) 322; Prog. Theor. Phys. 97 (1997) 901; S. M. Bilenky, C. Giunti and W. Grimus, Phys. Rev. D58 (1998) 033001; A. De Rujula, M. B. Gavela, and P. Hernandez, Nucl. Phys. B547 (1999) 21; K. Dick et al., Nucl. Phys. B562 (1999) 29; A. Gago, V. Pleitez and R. Zukanovich Funchal, Phys. Rev. D61 (2000) 016004 ; A. Donini et al., Nucl. Instrum. Meth. $\mathbf{A 4 5 1}$ (2000) 58.

10. S. P. Mikheyev and A. Smirnov, Nuovo Cim. 9C (1986) 17; L. Wolfenstein, Phys. Rev. D17 (1978) 2369.

11. J.N. Bahcall, P.I. Krastev and A.Yu. Smirnov, Phys. Rev. D60 (1999) 093001; M.C. Gonzalez-Garcia, P.C. de Holanda, C. Peña-Garay and J.W.F. Valle, Nucl. Phys. B573 (2000) 3.

12. G. L. Fogli, E. Lisi, D. Montanino, and A. Palazzo, Phys. Rev. D62 (2000) 013002.

13. C. Jarlskog, Phys. Rev. Lett. 55 (1985) 1039.

14. M. Koike and J. Sato, Phys. Rev. D61 (2000) 073012; hep-ph/9911258.

15. O. Yasuda, Acta. Phys. Polon. B 30 (1999) 3089 hep-ph/9910428.

16. CHOOZ Collaboration, M. Apollonio et al., Phys. Lett. B420 (1998) 397; ibid., B466 (1999) 415.

17. K. Nishikawa et al., Proposal for a Long Baseline Neutrino Oscillation Experiment using KEK-PS and SuperKamiokande, 1995; Y. Oyama, hep-ex/9803014.

18. Y. Mori, Talk at KEK International Workshop on "High Intensity Muon Sources", KEK, Japan, December 1-4, 1999, to appear in Proceedings.

19. W. Haxton, Phys. Rev. D36 (1987) 2283.

20. K. Nakamura, Talk at Workshop on Neutrino Oscillations and Their Origin, Fujiyoshida, Japan, Feb. 11-13, 2000, to appear in Proceedings.

21. Y. Kuno, in Proceedings of the Workshop on High Intensity Secondary Beam with Phase Rotation, September 21-22, 1998 (Institute for Chemical Research, Kyoto University). 